\title{
From a Desktop Application to a Web API A Code Conversion Approach
}

\author{
João Nuno Brito \\ Information Systems Engineering and Management \\ University of Minho \\ Guimarães, Portugal \\ A73310@alunos.uminho.pt
}

\author{
José Luís Pereira \\ Information Systems Department \& Algoritmi Center \\ University of Minho \\ Guimarães, Portugal \\ jlmp@dsi.uminho.pt
}

\begin{abstract}
Nowadays, hybrid solutions that manage the interaction between desktop platforms and Web applications and mobile devices are steadily gaining more and more importance. In this context, the use of Web services stands as the development technology to use. In this paper an automatable approach for the conversion of code from a desktop application to a Web service is presented. In order to demonstrate this approach, a real-life based scenario is presented, in which the services of an ERP (Enterprise Resource Planning) system were made available, automatically, via Web services, using the Web API (Application Programming Interface) framework from Microsoft.
\end{abstract}

\section{CCS Concepts}

-Software and its engineering $\rightarrow$ API languages

\section{Keywords}

Code conversion; REST; SOAP; Web API; ERP

\section{INTRODUCTION}

Nowadays, hybrid solutions that manage the interaction between desktop platforms and Web applications and mobile devices are steadily gaining more and more importance. The work described in this paper was originated from a real-life situation in which there was a need to connect a large and complex ERP system to the Web, giving its users a newer and more versatile way to access this platform. Frequently, over 200 users might access, at the same time, the services provided by the ERP system. Thus, scalability and low response times are important qualities to preserve. The ERP system itself is also evolving, with changes happening in a almost daily basis. Therefore, the manual implementation of a Web API was not an option, considering the vast amount of human resources needed to keep up with the changes being done. Thus, the need for an automated solution.

From the various approaches available currently to create a solution for this scenario, REST was chosen, mostly because of its ability to scale, its low response times and also its loosely coupling

Permission to make digital or hard copies of all or part of this work for personal or classroom use is granted without fee provided that copies are not made or distributed for profit or commercial advantage and that copies bear this notice and the full citation on the first page. Copyrights for components of this work owned by others than ACM must be honored. Abstracting with credit is permitted. To copy otherwise, or republish, to post on servers or to redistribute to lists, requires prior specific permission and/or a fee.

Request permissions from Permissions@acm.org.

ICGDA 2019, March 15-17, 2019, Prague, Czech Republic (C) 2019 Association for Computing Machinery. ACM ISBN 978-1-4503-6245-0/19/03...\$15.00

DOI: https://doi.org/10.1145/3318236.3318253 client-server quality, which allows the development of newer versions of the API, independently from the client. These characteristics are further explained in the second section of this paper, together with a brief comparison with SOAP, to further justify the selection made.

Based on the experience of creating solutions for the automatic conversion of code, a simple approach emerged, which is described in the third section of this paper. The code conversion approach is, mainly, a set of rules, steps, and guidelines that, although having the Web API framework from Microsoft in mind, should still apply in other cases.

The solution created to support the automation of code conversion from a desktop application to a Web API is described in the fourth section of this paper. In this section, some of the problems found during the development of the solution are also mentioned, as well as some future steps.

\section{RESTFUL WEB SERVICE}

Web services have been recognized as a standard for distributed computing and are considered, as a powerful and viable way to achieve universal interoperability [1].

In the software industry, it has been common for companies to try to win customers via proprietary platforms and technologies. Web services are a recent computing paradigm which is being supported by major software developers like IBM, Sun, and Microsoft, who traditionally would compete with their own technologies [2].

One of the multiple ways to develop a Web service is REST. REST is a newer approach (if compared to SOAP), which relies on the HTTP protocol to transmit data in multiple formats, such as XML, JSON, etc. [4]. It defines a group of constraints that, if followed, will result in a Web service that is focused on the system's resources, including how those resources' states are addressed and transferred over HTTP, by a wide range of clients [5].

REST provides its services through an initial URL (Uniform Resource Location), and the navigation is done according to the business logic, present in each use case, promoting a dynamic way to build routes that answer to the needs of each application [6].

According to Fielding [7], to create a RESTful Web service some constraints/characteristics must be taken into consideration. These are briefly discussed below.

A client-server architecture is, currently, the most common architecture for network communication, and its popularity can be perceived through the phenomenon that is the expansion of the World Wide Web. A dictionary-like definition for a client is a system or program that does requests to other systems or programs, called servers, to execute certain tasks; a server is a system or 
program which receives a request from a client and, consequently, performs a task to support the client's request and provide it with a response [8].

The communication between the client and the server must be stateless, as stated by Fielding [7]. Meaning all necessary information must be passed in each request from a client to a server. The server must understand the request without relying on stored context and the client must hold the entirety of the session state information.

Statelessness may decrease the network performance due to the repetition of data transfer across multiple requests. This issue can be mitigated by implementing a cache system, which is a way to improve the network efficiency. If a client receives data labelled as cacheable, the client can store it and use it later, for equivalent requests, saving time while communicating with the server. The downside of implementing cache is the risk of having unreliable data, in cases where the client has outdated data stored.

A uniform interface is one of the key differences between REST and the other approaches. Through applying the principle of software engineering generalization to the interface (contract between client and server) component, the system's architecture becomes more intuitive and the visibility of every available interaction is improved. This constraint has advantages and disadvantages, as it is optimized for generic cases, but, in some specific cases, it can become inefficient.

Fielding [7] describes a layered system as a way to improve security, organization, and scalability, by creating a hierarchy between layers, restricting the access of the non-adjacent layers.

Lastly, as described by Moro, Dorneles \& Rebonatto [9], the codeon-demand is an optional constraint, consisting on allowing the clients to be able to download and execute code directly from the client side. This way, the extensibility is improved, meaning that some features do not need to be pre-implemented, as they can be acquired later by the client. This constraint comes with the disadvantage of reducing the visibility, thus being optional.

As stated by Muehlen, Nickerson, and Swenson [10], it is clear that a piece is missing on the Web. Although human-to-machine communication works well, machine-to-machine communication is not as well developed and can be proven quite inconvenient. Web services such as REST and SOAP were originated to help to solve this issue, both in their own unique way.

These two approaches handle system interactions quite differently. SOAP is a protocol used for constructing and processing messages independently from the technological abilities of the receiver and can make use of different application layer protocols such as RPC (Remote Procedure Call), HTTP (Hypertext Transfer Protocol) or SMTP (Simple Mail Transfer Protocol). Instead, REST is a group of constraints designed for Web applications (making use of the HTTP) [11].

Tihomirovs and Grabis [12] argue that, in cases where scalability, compatibility, and performance are key requirements, REST is the best approach, as its implementation complexity, execution speed, consumed memory resources and performance are better in comparison to the SOAP protocol (see Figure 1). On the other hand, for cases where higher levels of security, readability, maintainability and lower number of possible errors on the client side are required, SOAP proves to be the best approach. SOAP also takes the lead in scenarios where asynchronous data processing requests are needed. In sum, SOAP should be the best option when dealing with large information systems integration efforts such as a banking information system.

\section{CODE CONVERSION APPROACH}

In this section, a simple approach for converting the code from a desktop application to a Web API is described. Though this approach was created with the Asp.NET Web API framework in mind, most steps should still apply to other code conversion scenarios.

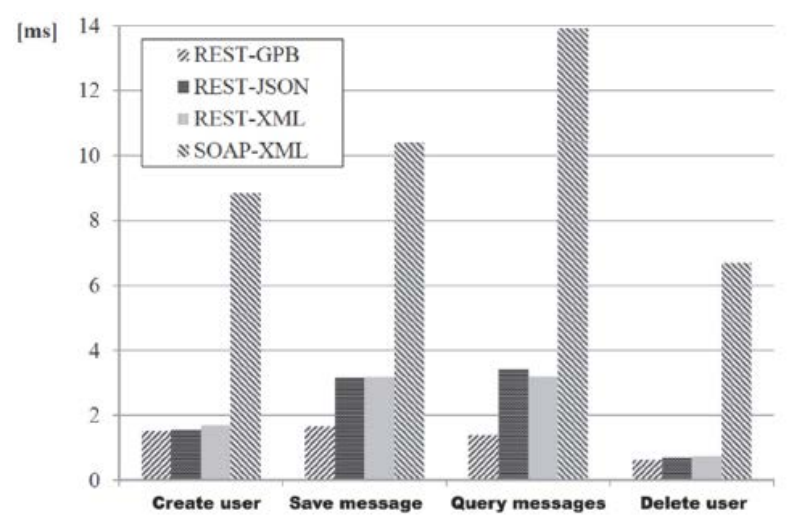

Figure 1. Response time - REST vs SOAP [12].

In order to succeed in the conversion of code from a desktop application to a Web API, it is required to understand both the starting point (desktop application) and the finish point (Web API). The most advisable way to convert the code is by isolating each desktop part and understanding where and how it will be reflected in the Web API. For example, each desktop class will be turned into a Web API controller with a reflection of its methods and arguments.

Although not mandatory, it is recommended to use attributed routing in a format similar to (1), below. This facilitates the usability, documentation, readability and significantly benefits the automation of the Web API controllers' creation. The uniform interface constraint is supported by the Web API with the attributed routing feature. This feature allows the controller to have a route prefix (see (2), below) and each method its own custom route (see (3) below), providing a good environment for developing a proper automatable uniform interface.

$$
\begin{aligned}
& \text { “\{URL }\} / \text { api } /\{\text { class }\} /\{\text { method }\} /\{\text { parameters }\} ” \\
& \text { “/api/\{class }\} ” \\
& \text { “/\{method }\} / \text { parameters\}” }
\end{aligned}
$$

After the classes are converted to controllers, they must be populated with the respective methods. Each method and each scenario are different, but the following guidelines should apply to most cases:

- $\quad$ There's only need for GET and POST type methods;

- All methods should be of the type GET unless:

- One or more complex objects are passed as arguments;

- Sensitive data needs to be used as input;

- The method changes the state of the application (e.g. creating a new user);

- If the type is GET, attributes should be passed in through attribute routing, opposed to convention-based routing;

- If a desktop method contains arguments by reference, they must not be reflected in the controller's method, only when calling the desktop method inside the controller's method; 
- If the method is dynamic, the result must be casted before being delivered to the client (explained in the following section).

The process of creating the controllers and populating them with methods can (and probably should) be automated. If the classes in the desktop application are properly built and have a good interface, any class can be read, followed by the iteration through its methods and the method's parameters. After having access to each class, method, and parameter individually, all that is left is the conversion into the Web API equivalent, which will depend on each case (Figure 2).

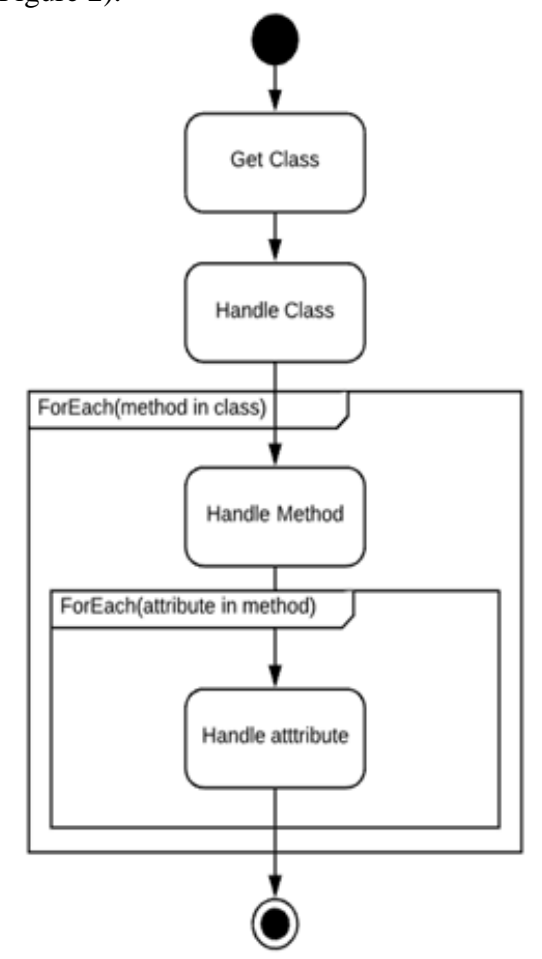

Figure 2. Generic code conversion flow.

\section{USING THE APPROACH IN A REAL CASE}

The work described in this section was originated from a need to automate as much as possible, the creation and maintenance of a Web service. This Web service would provide an interface between the Web and a desktop application, which consists of an ERP system, developed over many years, by many different people, reaching such a level of complexity and size, that the manual creation of such interface would be a poor use of valuable human resources.

The ERP system in question possesses a business service layer which allows the connection between the Web service and its different modules (e.g. Accounting, Sales, Treasury, etc.). This way, the Web service can call the ERP's methods and return the results to a client, following the business rules and keeping the data's integrity.

In this particular case, there is a great desire for scalability, as in some of the ERP's use cases, there are over 200 users accessing the ERP simultaneously. Therefore, the interface was made using a REST approach, rather than using SOAP.
An Asp.NET Web API is a framework created by Microsoft to facilitate the development of RESTful applications on the .NET framework. Although this paper has this framework in mind, most key ideas can still be functional for other scenarios.

\subsection{Web API}

In order to generate the most amount of code possible and to accommodate the module-based ERP, a core project with the Web API was created, alongside multiple Class Libraries referenced to it, with the purpose of containing each module. This way, features like caching and authentication can be managed in a single place, and the modules are isolated, providing a good environment for developing new modules and/or updating the already existing ones.

Nowadays there are many ways to develop an authentication system. Depending on each case, a different approach can be taken. The most common ones use OAuth, JWT (JSON Web Token) or SAML (Security Assertion Markup Language). In this case, OAuth was selected as opaque tokens are made for situations where only one entity needs to validate the tokens and there is no need for the token owner to examine its claims.

Even though caching is optional, it is worthwhile to include since it significantly increases the performance of the Web API. Caching is not built into ASP.NET Web API, so an appropriate solution must be found to each case. Some of the existing open source projects currently available include "CacheCow" 1 and "Strathweb.CacheOutput" 2 . There are also the following namespaces: "System.Runtime.Caching" 3 and "System.Web.HttpRuntime.Cache" ${ }^{4}$. Finally, Redis ${ }^{5}$, an open source in-memory data structure store that can be used as a database, cache and message broker, can also be used.

To access the ERP's resources, the user must begin by authenticating her/himself, thus entering her/his credentials on the website, which will then call the Web API, followed by the authorization server, followed by the ERP. In case the credentials are valid, the authorization server will generate a token, and it will be stored in the client's cache for later usage. After obtaining the token, the user can access the resources in the ERP. The user can then send requests to the Web API with the token in the request's header, along with the necessary information to access the method. The Web API validates the token with the authorization server and calls the matching method from the ERP, returning to the client the result of the method.

\subsection{Code Generator}

The ERP is a large software system under continuous development. The creation of a static Web API solution that would require constant maintenance to keep up with the changes made to the application would result in a considerable amount of wasted resources. Thus, it is proposed to automate most of the creation and management of the Web API.

In order to achieve a solution, it is recommended to begin with the creation of working prototypes of a few specific controllers with methods connecting to the application. This way, there is a target used to compare the code generator's output.

${ }^{1}$ https://github.com/aliostad/CacheCow

${ }^{2}$ https://github.com/filipw/Strathweb.CacheOutput

${ }^{3}$ https://msdn.microsoft.com/enus/library/system.runtime.caching(v=vs.110).aspx

${ }^{4}$ https://msdn.microsoft.com/enus/library/system.web.httpruntime.cache(v=vs.110).aspx

${ }^{5}$ https://redis.io/ 
Despite the many ways available to create a file, read and write to it, the open source "PrimaveraDarwin/Darwin" ${ }^{6}$ project was used as a base for the code generator. One of this project's features is to read DLLs and output unit tests for the ERP. This feature can be adapted in order to output Web API controllers, based on each class existent in a DLL.

After the necessary changes, the interface had the two required fields: source (path to the input DLL) and destination (output path where classes will be generated to). Other fields were added to handle a blacklist for a group of methods existing in the desktop version of the application, not required on the Web.

The activity diagram presented in Figure 3 represents the activities executed while running the code generator program, starting by checking the paths of the source and destination, making sure there is a DLL to read from and a folder to output the generated files. Afterward, the module is identified and an iteration through its submodules starts. For each submodule, there is a checkpoint to validate if the submodule's file should be created and, in a positive instance, the class header (references, namespace, class name and controller route) is written. Next, for each method in the submodule, there is a validation and, if positive, the method is written. Finally, after iterating through all the submodules, the file and method count is delivered to the user interface and the program stops.

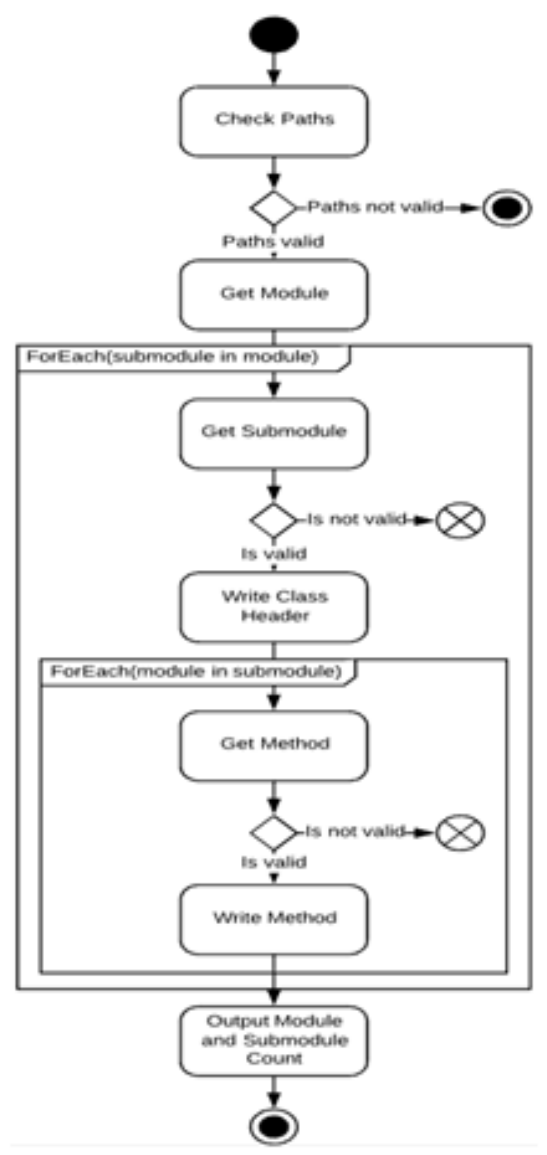

Figure 3. Code generation activity diagram.

In case of multiple complex objects being passed through the method as attributes, a solution must be created. For that, the

\footnotetext{
${ }^{6}$ https://github.com/PrimaveraDarwin/darwin/
}

JObject class was used. It can receive a JSON with the multiple objects, which can be separated and handled individually later.

In case attributes were passed by reference, an exception was added to remove them from the Web API's call and handled inside the method. For cases like this, a response protocol was created in which, if variables by reference existed in the call, instead of the regular desktop method's return, a JSON would be returned with a specific format. There are also cases in which the desktop method might be dynamic, so it must be casted before the API's return call.

Figure 4 (below) represents a generic example of a generated method with the code generator.

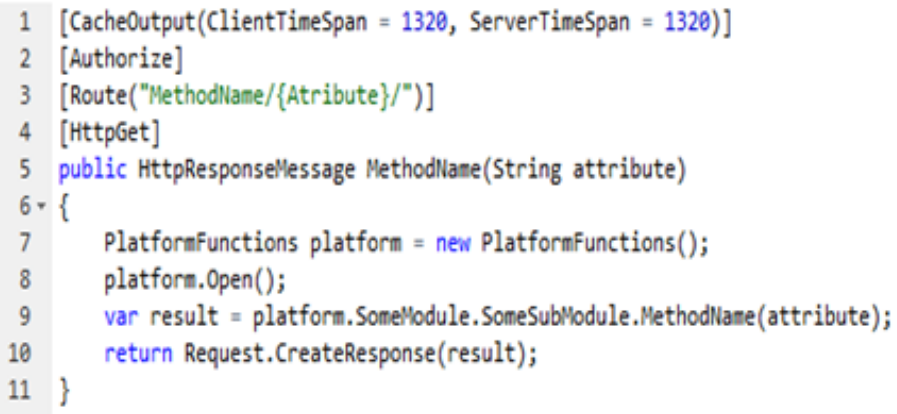

Figure 4. Generic method example.

The key parts of a method are the following:

- $\quad$ Line 1: Cache management. In GET methods, the result is stored in the client's cache using the open source project "Strathweb.CacheOutput";

- $\quad$ Line 2: Authorization. This line forces the client to send a valid token in the request header to be able to access the resource. This was possible, using OAuth2.0;

- $\quad$ Line 3: Route. In order to generate code in a logical way, the method's route is always the method name followed by its attributes, and in case there is an object, it is passed through the body;

- $\quad$ Lines 7 and 8: Declaring and opening the platform variable. In this particular case, in order to access the ERP's methods, there is a need to open the ERP's platform;

- $\quad$ Line 9: Calling the ERP's method;

- Line 10: Returning the value.

As it usually happens in every development project, there were some setbacks in the process of developing this solution. One of the most time consuming was the process of going through the 320 DLLs present in the ERP's solution and adding only the necessary ones to the Web API project, to avoid overloading the Web API with un-used files. This whole process was valuable to save time in every code compilation done in the future.

Another example would be dealing with the continuous development of the ERP, which in some cases stopped the Web API's functionality completely. This issue was originated by the “distance” between the ERP's and Web API's developer teams. It is crucial for every project that all teams involved in the process are constantly aware of the ideas and constraints of each other.

This solution is still under development, as some details must be polished, and the overall performance can be improved in a further stage where both the ERP system teams and the Web API team come together to construct a better and most efficient way for the ERP system to work, fitting both teams’ plans. 


\section{CONCLUSIONS}

The major contribution of this paper is a simple approach that provides a walkthrough with steps, rules, and guidelines that should be followed for the successful conversion of a desktop's application code to a functional REST Web service. It is important to note that most principles still apply to create other REST Web services which are not based on the Web API framework. Although easily automatable, this approach was meant to supply to most use cases where the applications are smaller sized and don't require as much maintenance.

To demonstrate its applicability a real-life scenario usage of the code conversion approach was presented. In this scenario, there was a need to connect a large ERP system to the Web, providing its users with a new and more flexible way of accessing the information in the desktop application. Developing a Web service seemed like the suitable answer, but it would not be feasible if done manually, as the ERP system has been, and continues to be, under constant development for over 20 years, by multiple teams. It would require similar sized teams to keep up with the development of the program and doubling the human resource needs was not worth the new feature. So, it was considered pertinent to develop an automated way to create and manage the Web API. The project was divided into two separate solutions, one with the Web API (that would act as the foundation), and another one for the automatic generation of code (that would generate each of the ERP's modules individually). This division, along with proper documentation, allowed great flexibility for enabling and disabling specific modules, according to specifications.

Although functional, the solution presented is still not fully tested and so, it is susceptible to changes. Nevertheless, four of the ERP's modules were already successfully generated and implemented, with a total of around 300 classes and almost 5000 methods. Due to its caching feature, the solution is also able to have response times of less than 10 milliseconds, which is a good starting point, as some of the response times might have to be sacrificed in favour of stability. All in all, the approach presented is this paper has proved its value and practical interest.

\section{ACKNOWLEDGMENT}

This work has been supported by Primavera Business Software Solutions.

\section{REFERENCES}

[1] Zhao, J. L., \& Cheng, H. K. (2005). Web services and process management: a union of convenience or a new area of research? Decision Support Systems, 40(1), 1-8.

[2] Tang, Q. C., \& Cheng, H. K. (2005). Optimal location and pricing of web services intermediary. Decision Support Systems, 40(1), 129-141.

[3] Serrano, N., Hernantes, J., \& Gallardo, G. (2014). Serviceoriented architecture and legacy systems. IEEE software, 31(5), 15-19.

[4] Rodriguez, A. (2008). Restful web services: The basics. IBM developerWorks, 33.

[5] Ribeiro, M. F., \& Francisco, R. E. (2016). Web Services REST: Conceitos, Análise e Implementação. Educação, Tecnologia e Cultura-ETC, (14).

[6] Fielding, R. T., \& Taylor, R. N. (2000). Architectural styles and the design of network-based software architectures (Vol. 7). Doctoral dissertation: University of California, Irvine.

[7] Hanson, M. D. (2000). The Client/Server Architecture. Server Management, 3.

[8] Moro, T., Dorneles, C., \& Rebonatto, M. T. (2009). Web services WS-* versus Web Services REST. Revista de Iniciação Científica, 11(1).

[9] Zur Muehlen, M., Nickerson, J. V., \& Swenson, K. D. (2005). Developing web services choreography standards - the case of REST vs. SOAP. Decision Support Systems, 40(1), 9-29.

[10] Potti, P. K., Ahuja, S., Umapathy, K., \& Prodanoff, Z. (2012). Comparing performance of web service interaction styles: Soap vs. rest. In Proceedings of the Conference on Information Systems Applied Research ISSN (Vol. 2167, p. 1508).

[11] Tihomirovs, J., \& Grabis, J. (2016). Comparison of soap and rest based web services using software evaluation metrics. Information Technology and Management Science, 19(1), 9297. 